\title{
Research Communication Serum Adipokine and Ghrelin Levels in Nonalcoholic Steatohepatitis
} \author{
Orhan K. Poyrazoglu, ${ }^{1}$ and Ahmet Erensoy ${ }^{4}$ \\ ${ }^{1}$ Department of Gastroenterology, School of Medicine, Firat University, 23200 Elazig, Turkey \\ ${ }^{2}$ Department of Biochemistry, School of Medicine, Firat University, 23200 Elazig, Turkey \\ ${ }^{3}$ Department of Immunology, School of Medicine, Firat University, 23200 Elazig, Turkey \\ ${ }^{4}$ Department of Microbiology, School of Medicine, Firat University, 23200 Elazig, Turkey
}

Mehmet Yalniz, ${ }^{1}$ Ibrahim Halil Bahcecioglu, ${ }^{1}$ Huseyin Ataseven, ${ }^{1}$ Bilal Ustundag, ${ }^{2}$ Fulya Ilhan, ${ }^{3}$

Received 22 May 2006; Revised 23 August 2006; Accepted 23 August 2006

\begin{abstract}
Adipokines and ghrelin play role in insulin resistance, the key pathophysiological abnormality in patients with nonalcoholic fatty liver diseases. In the present study, relationship between nonalcoholic steatohepatitis (NASH) and serum adipokine and ghrelin levels was investigated. Thirty seven patients with biopsy-proven NASH and 25 age- and sex-matched controls were enrolled. Ten of NASH patients $(27 \%)$ had diabetes mellitus $(n=5)$ or impaired glucose tolerance $(n=5)$. Body mass index (BMI) was less than $30 \mathrm{~kg} / \mathrm{m}^{2}$ in $67.6 \%$ of patients, while in the remaining $32.4 \%$ it was more than $30 \mathrm{~kg} / \mathrm{m}^{2}$. Serum adiponectin, leptin, TNF$\alpha$, and ghrelin were determined. Serum leptin $(15.49 \pm 4.84$ vs $10.31 \pm 2.53)$ and TNF- $\alpha(12.1 \pm 2.7$ vs $10.31 \pm 2.56)$ levels were significantly higher in the NASH group compared to in the control group $(P<.001$ for each). Nevertheless, adiponectin $(11.1 \pm 2.1$ vs $17.3 \pm 2.8)$ and ghrelin $(6.46 \pm 1.1$ vs $7.8 \pm 1.1)$ levels were lower in the NASH group than in the control group $(P<.001$ for each). Serum levels of the adipokines and ghrelin, however, were comparable in the subgroups of patients regardless of whether BMI was $<30$ or $>30$ or glucose tolerance was impaired or not $(P>.05)$. Additionally, neither adipokines nor ghrelin was correlated with histopathological grade and stage $(P>.05)$. In conclusion; there is a significant relationship between NASH and adipokines and ghrelin independent from BMI and status of the glucose metabolism. These cytokines that appear to have role in the pathogenesis of NASH, however, do not have any effect upon the severity of the histopathology.
\end{abstract}

Copyright @ 2006 Mehmet Yalniz et al. This is an open access article distributed under the Creative Commons Attribution License, which permits unrestricted use, distribution, and reproduction in any medium, provided the original work is properly cited.

\section{INTRODUCTION}

It has been well established that nonalcoholic fatty liver (NAFLD) is intimately related to various clinical and biological markers of the insulin resistance syndrome [1]. Furthermore, there is a near-universal association between nonalcoholic steatohepatitis (NASH), the severe and progressive form of NAFLD, and insulin resistance, irrespective of obesity [2].

Adipose tissue has currently been regarded as an active player in the regulation of metabolism since the discovery of several adipocyte-derived factors, collectively known as adipokines [3]. In this context, numerous substances, mainly released by adipose tissue including tumor necrosis factor-alpha (TNF- $\alpha$ ), leptin, and adiponectin, are closely linked to each other and are thought to contribute to peripheral insulin resistance [4-7]. In addition, ghrelin, a novel peptide isolated from human and rat stomach, is also associated with insulin resistance beside its role in glucose and energy hemostasis and food intake [8].

Adipokines and ghrelin have been implicated in the pathogenesis of NAFLD through their metabolic and/or pro-/anti-inflammatory activity [9-12]. Despite the emerging data showing an association between NASH and the aforementioned substances, existing results, however, are still controversial. One reason for this discrepancy among the studies is the disparity of the methods used in the studies as well as the inhomogeneous feature of the study populations. Secondly, these parameters are determined separately in most of the studies. Only in two recent studies $[13,14]$, adiponectin, TNF- $\alpha$, and leptin were collectively analyzed in the same study population. These two studies, however, have also yielded conflicting results. The only consistent result of the two studies was the association between hypoadiponectinemia and NASH. 
In the present study, serum adipocytokines and, additionally, ghrelin levels were determined in patients with biopsy-proven NASH.

\section{METHODS}

Thirty seven patients with histopathologically proven NASH and age- and sex- matched twenty five healthy individuals were enrolled to the study.

Informed consent was obtained from each patient and the study protocol conforms to the ethical guidelines of the 1975 Declaration of Helsinki as reflected in a priori approval by the institution's human research committee. Local ethic committee approved the study.

Control group consisted in individuals who had normal abdominal ultrasound liver scans, normal transaminase values, normal fasting serum glucose levels, and normal glucose tolerance tests.

The diagnosis of NASH was based on the following criteria: (1) the presence of steatosis (>10\%), lobular inflammation, and ballooning degeneration (with or without fibrosis) on liver biopsy; (2) the intake of less than $20 \mathrm{~g}$ of ethanol per day, as confirmed by the physician and family members who were in close contact with the patient; and (3) appropriate exclusion of other liver diseases such as alcoholic liver disease, viral hepatitis, autoimmune hepatitis, drug-induced liver disease, primary biliary cirrhosis, primary sclerosing cholangitis, biliary obstruction, celiac disease, and metabolic liver diseases. No patient had a history of jejunoileal bypass.

Histopathological grading and staging of the NASH was made according to Brunt's criteria [15] by a specialist pathologist, who also made the histopathological diagnosis of the NASH.

\section{Grading}

Grading was made according to macrovesicular steatosis and necroinflammatory activity.

Macrovesicular steatosis. Grade 0: no steatosis; Grade 1: steatosis up to 33\%; Grade 2: steatosis between 33 and $66 \%$; Grade 3: steatosis over 66\%.

Necroinflammatory activity. Grade 1: mild; Grade 2: moderate; Grade 3: severe.

\section{Staging}

Staging was made according to fibrosis.

Stage 1: zone 3 perisinusoidal/pericellular fibrosis; focal or diffuse. Stage 2: focal or diffuse periportal fibrosis together with zone 3 perisinusoidal/pericellular fibrosis. Stage 3: focal and diffuse bridging necrosis together with perisinusoidal/pericellular fibrosis and portal fibrosis. Stage 4: cirrhosis.

\section{Laboratory Analyses}

Blood samples were collected from patients and control group after an overnight fasting. Aspartate and alanine ami- notransferases (AST, ALT), total protein, albumin, alkaline phosphatase, $\gamma$-glutamyl transpeptidase, HbsAg, antiHCV, smooth muscle antibody, antimitochondrial antibody, serum cholesterol, triglyceride, fasting glucose levels, insulin, C-peptide, and complete blood count were studied.

Patients with fasting serum glucose levels of more than $126 \mathrm{mg} / \mathrm{dL}$ in at least two separate samples were identified as having diabetes mellitus, and a finding of $140-200 \mathrm{mg} / \mathrm{dL}$ two hours after the standard oral glucose loading was considered abnormal glucose tolerance test.

Homeostasis (HOMA) model was used for estimating the insulin resistance (IR).

HOMA IR $(\%)$ = fasting blood glucose $(\mathrm{mg} / \mathrm{dL}) /$ $18 \times$ fasting insulin $(\mu \mathrm{U} / \mathrm{mL}) / 22.5$.

As a measure of overweightness and/or obesity, body mass index (BMI) was calculated as the weight $(\mathrm{kg})$ divided by the square of height $\left(\mathrm{m}^{2}\right)$ in all participants and the patients with BMI of more than 30 were considered to show manifest obesity according to the World Health Organization classification [16].

\section{Analyses of serum adipokines and ghrelin}

Serum samples were taken from the patients and healthy individuals after an overnight fasting and kept in $-30^{\circ} \mathrm{C}$ until analyzed. TNF- $\alpha$, leptin, adiponectin, and ghrelin levels were analyzed with ELISA kits (TNF- $\alpha$ kit (range of detection: $0.5-32 \mathrm{pg} / \mathrm{mL}$, sensitivity: $<=0.09 \mathrm{pg} / \mathrm{mL}$ ) was purchased from BioSource International Inc, 542, Flynn Road, Camarillo, Calif, USA; ghrelin kit (range of detection: 0$100 \mathrm{ng} / \mathrm{mL}$, sensitivity: $0.08 \mathrm{ng} / \mathrm{mL}$ ) from Phoenix International; adiponectin kit (range of detection: $0.23-15 \mathrm{ng} / \mathrm{mL}$, sensitivity: $0.1 \mathrm{ng} / \mathrm{mL}$ ) from Chemicon International, Inc, USA; and leptin kit (sensitivity: $0.2 \mathrm{ng} / \mathrm{mL}$ ) from DRG International, Inc, USA).

Levels of adipokines and ghrelin are closely related to body fat mass and may simply reflect the association between NAFLD and other risk factors for liver diseases like obesity and diabetes rather than a true causal relationship. Hence, patients are divided into subgroups according to the BMI and glucose metabolism status, in order to determine the adipokines and ghrelin in study populations with different BMI and glucose metabolism that might have impact upon the levels of these parameters.

\section{Statistical analysis}

All analyses were performed using the Statistical Package for the Social Sciences (SPSS) for Windows, version 11,0 packed program. Data were presented as mean \pm standard deviation unless noted as different. Difference between the groups was analyzed using Mann-Whitney U test. The relationships among the variables were analyzed using Spearman correlation test. $P<0.05$ was considered statistically significant. 
TABle 1: Demographic and clinical data in control and NASH groups.

\begin{tabular}{l|ccc}
\hline Parameter & $\begin{array}{c}\text { Control } \\
(n=25)\end{array}$ & $\begin{array}{c}\text { NASH } \\
(n=37)\end{array}$ & $P$ \\
\hline Age (years) & $37.1 \pm 7.75$ & $40.1 \pm 8.1$ & $>.05$ \\
Male/female (n) & $10 / 15$ & $25 / 12$ & $>.05$ \\
AST (IU/L) & $19.6 \pm 6.2$ & $60.6 \pm 34.6$ & $<.001$ \\
ALT (IU/L) & $19.4 \pm 4.5$ & $114.9 \pm 87.9$ & $<.001$ \\
Glucose (mg/dL) & $88.1 \pm 24.7$ & $113 \pm 44.9$ & $<.05$ \\
Cholesterol (mg/dL) & $181.0 \pm 33.1$ & $202.7 \pm 41$ & $>.05$ \\
Triglyceride (mg/dL) & $119.7 \pm 65.8$ & $225.7 \pm 112$ & $<.001$ \\
Impaired GT*/DM (n) & $0 / 0$ & $5 / 5$ & $<.001$ \\
BMI (kg/m $\left.{ }^{2}\right)$ & $24.1 \pm 1.9$ & $28.7 \pm 3.3$ & $<.05$ \\
HOMA index & $1.75 \pm 0.67$ & $7.0 \pm 9.2$ & $<.001$ \\
\hline
\end{tabular}

${ }^{*} \mathrm{GT}$ : glucose tolerance.

\section{RESULTS}

Demographic and clinical data of the patients and the control subjects are shown in Table 1. Mean age and gender between the control and NASH groups were comparable $(P>0.05)$. On the other hand, mean BMI and HOMA IR were significantly higher in the NASH group than in the control group $(P<0.05$ and 0.001 , resp). Control subjects had neither impaired glucose tolerance nor diabetes mellitus. Nonetheless, ten $(27.03 \%)$ of the thirty seven NASH patients had abnormal glucose metabolism, five had impaired glucose tolerance, and five had diabetes mellitus.

In histopathological analysis, nine $(24.3 \%)$ of the 37 patients with NASH had grade 1, nineteen (51.4\%) had grade 2 , and nine $(24.3 \%)$ had grade 3 disease. Most of the patients $(n=23(62.2 \%))$ were in stage 2 histopathologically. The distribution of the remaining patients was as follows: seven $(18.9 \%)$ in stage 0 , one $(2.7 \%)$ in stage 1 , and six $(16.2 \%)$ in stage 3 disease.

\section{Serum adipokine and ghrelin levels}

Serum leptin and TNF- $\alpha$ levels were significantly higher in the NASH group compared to in the control group $(P<$ 0.001 for each). Nevertheless, adiponectin and ghrelin levels were found decreased in the NASH group than in the control group $(P<0.001$ for each). These differences, however, were not gender specific in the NASH group $(P>0.05)$. Serum levels of the adipokines and ghrelin were comparable in the subgroups of patients regardless to whether BMI was $<30$ or $>30$ or glucose tolerance was impaired or not $(P>0.05)$.

The values of the analyzed substances were also examined in different histopathological grade and stage of the NASH. Nevertheless, serum adipokine and ghrelin levels were not significantly different among the different grades or stages of the NASH (data not shown). On the other hand, patients with higher stages of NASH had higher HOMA IR values than patients with lower disease stages $(P<0.05)$.
Serum adipokine and ghrelin levels in the control group and patients with $\mathrm{NASH}$ and in patients with $\mathrm{NASH}$ are shown in Table 2.

\section{Relationship between the determined parameters and the histopathological findings}

A significant positive correlation was found only between ghrelin and TNF- $\alpha(r=0.491, P<0.05)$. Leptin had a positive correlation with BMI $(r=0.335, P<0.05)$ and a negative correlation with AST/ALT rate $(r=-0.328, P<$ $0.05)$. Neither adipokines nor ghrelin, however, correlated with histopathological grade and stage $(P>0.05)$.

\section{DISCUSSION}

The studies that evaluated serum adipokines separately yielded conflicting results although an association with NAFLD including NASH has been implicated. The results of two recent comprehensive studies $[13,14]$ that examined the adipokines (adiponectin, TNF- $\alpha$, and leptin) in patients with NASH are also at variance. The only consistent consequence of both studies was the association between hypoadiponectinemia and NASH, which is also in line with the present study. In addition, we found either TNF- $\alpha$ or leptin remarkably increased in patients with NASH. In light of these studies, it can be concluded that hypoadiponectinemia and the levels of other adipokines in NASH appear to be a part of a metabolic disturbance characterized by ectopic fat accumulation in the central compartment. The discrepancy among the results of these studies, however, appears to be related to the different nature of study populations; in particular, the study of Musso et al [14] which was consisted of nondiabetic and nonobese NAFLD patients and virtually of male gender $(92 \%)$.

In addition to adipokines, we examined serum ghrelin levels, too. This is the second study that examined ghrelin, a peptide intimately associated with insulin resistance; in patients with NASH. In keeping with the previous study [12], we found that serum ghrelin decreased in patients with NASH irrespective of BMI and the status of the glucose metabolism, the same as the adipokines. The observation that ghrelin levels are markedly reduced in patients with NASH might reflect a possible hepato-protective role of ghrelin similar to the adiponectin. If so, ghrelin-based future therapeutic trials of NAFLD might be appealing.

Serum levels of adipokines are related to body fat mass and diabetes. Hence, the presence of these confounding factors might hinder the interpretation of the association between adipokines and NAFLD. In this context, one of the remarkable findings of the present study is the values of the determined adipokines and ghrelin in patients with NASH were significantly different from the control group not only in patients with overt obesity $(\mathrm{BMI}>30)$, impaired glucose tolerance, or overt diabetes, but also in patients with BMI less than 30 or without impaired glucose tolerance or diabetes.

Moreover, although all of the examined substances are closely related with insulin resistance, neither adipokines nor 
TABLE 2: Data of the serum TNF- $\alpha$, leptin, adiponectin, and ghrelin values in control and NASH groups and in different subgroups of the NASH patients.

\begin{tabular}{|c|c|c|c|c|}
\hline Groups & TNF- $\alpha(\mathrm{pg} / \mathrm{mL})$ & Leptin $(\mathrm{ng} / \mathrm{mL})$ & Adiponectin (ng/mL) & Ghrelin $(\mathrm{ng} / \mathrm{mL})$ \\
\hline Control group $(n=25)$ & $3.35 \pm 1.2^{*}$ & $10.3 \pm 2.53^{*}$ & $17.3 \pm 2.8^{*}$ & $7.8 \pm 1.1^{*}$ \\
\hline NASH group $(n=37)$ & $12.1 \pm 2.7^{*}$ & $15.49 \pm 4.84^{*}$ & $11.1 \pm 2.1^{*}$ & $6.46 \pm 1.1^{*}$ \\
\hline Male $(n=25)$ & $11.9 \pm 2.8$ & $15.3 \pm 5.2$ & $11 \pm 2.3$ & $6.4 \pm 1.1$ \\
\hline Female $(n=12)$ & $12.3 \pm 2.5$ & $15.9 \pm 4.3$ & $11.3 \pm 1.9$ & $6.7 \pm 1.1$ \\
\hline $\mathrm{BMI}<30(n=25)$ & $12.1 \pm 2.8$ & $15.1 \pm 5.0$ & $11.1 \pm 2.1$ & $6.5 \pm 1.2$ \\
\hline $\mathrm{BMI}>30(n=12)$ & $12.0 \pm 2.5$ & $16.3 \pm 4.5$ & $11.1 \pm 2.3$ & $6.4 \pm 1.0$ \\
\hline Normal GT $(n=27)$ & $12.2 \pm 2.8$ & $15.4 \pm 5.3$ & $11 \pm 2.4$ & $6.4 \pm 1.1$ \\
\hline Impaired GT** and DM $(n=10)$ & $11.6 \pm 2.6$ & $15.9 \pm 3.5$ & $11.4 \pm 1.3$ & $6.7 \pm 1.2$ \\
\hline
\end{tabular}

**GT: glucose tolerance.

${ }^{*} P<.001$ between control and groups..

ghrelin is correlated with HOMA in the patient group indicating that this relation is independent from insulin resistance and BMI, in keeping with the previous studies [13]. Yamamoto et al [17] have reported that adiponectin predicts future insulin resistance in a two-year followup study. Hypoadiponectinemia, therefore, might well be an early predictor of overt diabetes and overall or central obesity that might emerge in patients with NAFLD in the future.

Hypoadiponectinemia has been found related with necro-inflammatory activity [13] and fibrosis [14]. Additionally, adiponectin has anti-inflammatory properties in the liver and its deficiency accounts for high aminotransferase values and disease progression. A direct antifibrotic effect of adiponectin has also been shown on the basis of expression of adiponectin gene in hepatic stellate cells [18]. Neither serum adipokine including adiponectin nor ghrelin, however, is found associated with the histopathological severity in the present study. This finding does not lend support to previously reported relationship between adiponectin levels and necro-inflammatory activity [13]. One explanation of this controversy might be the fluctuating pattern of serum adipokine and ghrelin levels over time depending on the metabolic milieu. Additionally, hepatic mRNA expression of adiponectin receptors (AdipoRII) in NASH inversely correlated with the histological grade of fibrosis but not with serum and hepatic adiponectin [19]. Hence, the necroinflammatory or fibrogenic action of adiponectin seems to be mostly depending on its intrahepatic production, ensuing in autocrine or paracrine actions, in a cytokine-like manner. Future studies are warranted to elicit the relative involvement of circulating versus locally produced adiponectin in the regulation of liver response to injury.

In conclusion, there is a significant relation between $\mathrm{NASH}$ and serum adipokine and ghrelin values irrespective of BMI and status of glucose metabolism. It seems that this relationship is, at least partly, independent from insulin resistance. Additionally, serum adipokine and ghrelin that have been ascribed to have role in the pathogenesis of NASH do not have any association with histopathological severity of the disease. This finding indicates that future studies incorporating tissue measures of the adipokines and ghrelin are required to elucidate the precise role of adipokines upon histopathological severity in NASH.

\section{ACKNOWLEDGMENT}

This work was supported by a grant from Firat University Scientific Research and Projects Section.

\section{REFERENCES}

[1] Luyckx FH, Lefebvre PJ, Scheen AJ. Non-alcoholic steatohepatitis: association with obesity and insulin resistance, and influence of weight loss. Diabetes and Metabolism. 2000;26(2): 98-106.

[2] Nakao K, Nakata K, Ohtsubo N, et al. Association between nonalcoholic fatty liver, markers of obesity, and serum leptin level in young adults. American Journal of Gastroenterology. 2002;97(7):1796-1801.

[3] Frühbeck G, Gómez-Ambrosi J, Muruzábal FJ, Burrell MA. The adipocyte: a model for integration of endocrine and metabolic signaling in energy metabolism regulation. American Journal of Physiology-Endocrinology and Metabolism. 2001; 280(6):E827-E847.

[4] Kahn BB, Flier JS. Obesity and insulin resistance. Journal of Clinical Investigation. 2000;106(4):473-481.

[5] Sethi JK, Hotamisligil GS. The role of TNF $\alpha$ in adipocyte metabolism. Seminars in Cell and Developmental Biology. 1999; 10(1):19-29.

[6] Holst D, Grimaldi PA. New factors in the regulation of adipose differentiation and metabolism. Current Opinion in Lipidology. 2002;13(3):241-245.

[7] Rajala MW, Scherer PE. Minireview: the adipocyte-at the crossroads of energy homeostasis, inflammation, and atherosclerosis. Endocrinology. 2003;144(9):3765-3773.

[8] Pagotto U, Gambineri A, Vicennati V, Heiman ML, Tschöp M, Pasquali R. Plasma ghrelin, obesity, and the polycystic ovary syndrome: correlation with insulin resistance and androgen levels. Journal of Clinical Endocrinology and Metabolism. 2002; 87(12):5625-5629. 
[9] Crespo J, Cayón A, Fernández-Gil P, et al. Gene expression of tumor necrosis factor $\alpha$ and TNF-receptors, p55 and $\mathrm{p} 75$, in nonalcoholic steatohepatitis patients. Hepatology. 2001;34(6):1158-1163.

[10] Chitturi S, Farrell G, Frost L, et al. Serum leptin in NASH correlates with hepatic steatosis but not fibrosis: a manifestation of lipotoxicity? Hepatology. 2002;36(2):403-409.

[11] Xu A, Wang Y, Keshaw H, Xu LY, Lam KSL, Cooper GJS. The fat-derived hormone adiponectin alleviates alcoholic and nonalcoholic fatty liver diseases in mice. Journal of Clinical Investigation. 2003;112(1):91-100.

[12] Marchesini G, Pagotto U, Bugianesi E, et al. Low ghrelin concentrations in nonalcoholic fatty liver disease are related to insulin resistance. The Journal of Clinical Endocrinology and Metabolism. 2003;88(12):5674-5679.

[13] Hui JM, Hodge A, Farrell GC, Kench JG, Kriketos A, George J. Beyond insulin resistance in NASH: TNF- $\alpha$ or adiponectin? Hepatology. 2004;40(1):46-54.

[14] Musso G, Gambino R, Durazzo M, et al. Adipokines in NASH: postprandial lipid metabolism as a link between adiponectin and liver disease. Hepatology. 2005;42(5):1175-1183.

[15] Brunt EM, Janney CG, Di Bisceglie AM, Neuschwander-Tetri BA, Bacon BR. Nonalcoholic steatohepatitis: a proposal for grading and staging the histological lesions. American Journal of Gastroenterology. 1999;94(9):2467-2474.

[16] Obesity: preventing and managing the global epidemic. Report of a WHO consultation. World Health Organization Technical Report Series. 2000;894:i-xii, 1-253.

[17] Yamamoto Y, Hirose H, Saito I, Nishikai K, Saruta T. Adiponectin, an adipocyte-derived protein, predicts future insulin resistance: two-year follow-up study in Japanese population. Journal of Clinical Endocrinology and Metabolism. 2004;89(1):87-90.

[18] Kamada Y, Tamura S, Kiso S, et al. Enhanced carbon tetrachloride-induced liver fibrosis in mice lacking adiponectin. Gastroenterology. 2003;125(6):1796-1807.

[19] Kaser S, Maschen A, Cayon A, et al. Adiponectin and its receptors in non-alcoholic steatohepatitis. Gut. 2005;54(1):117121. 


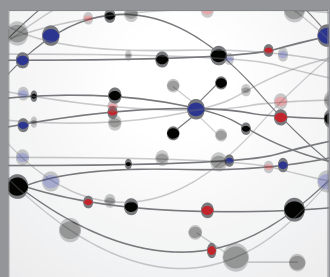

The Scientific World Journal
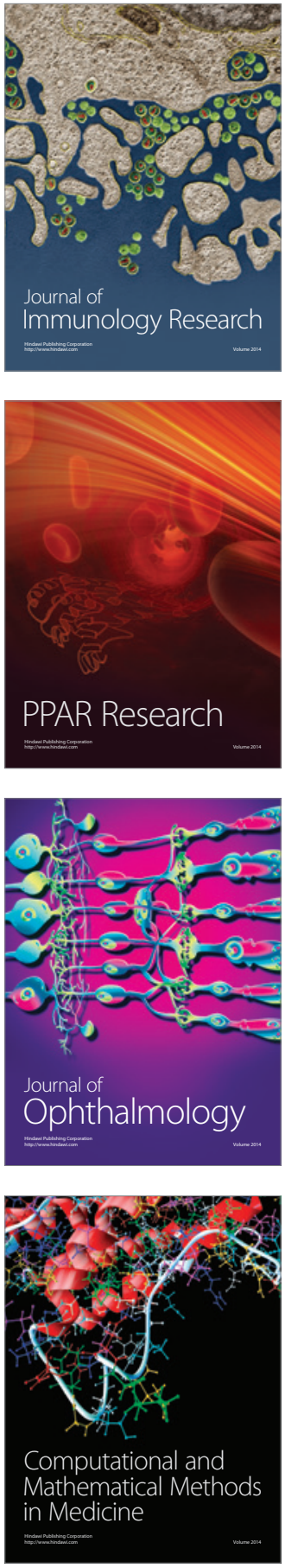

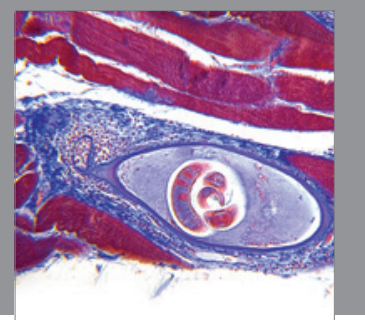

Gastroenterology

Research and Practice
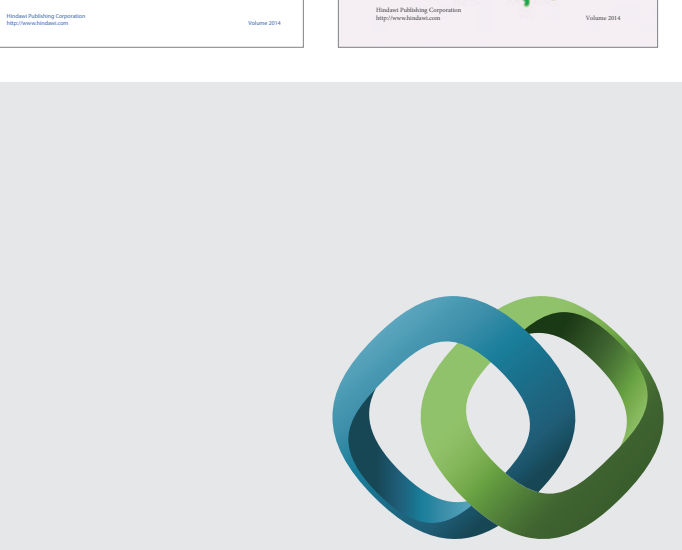

\section{Hindawi}

Submit your manuscripts at

http://www.hindawi.com
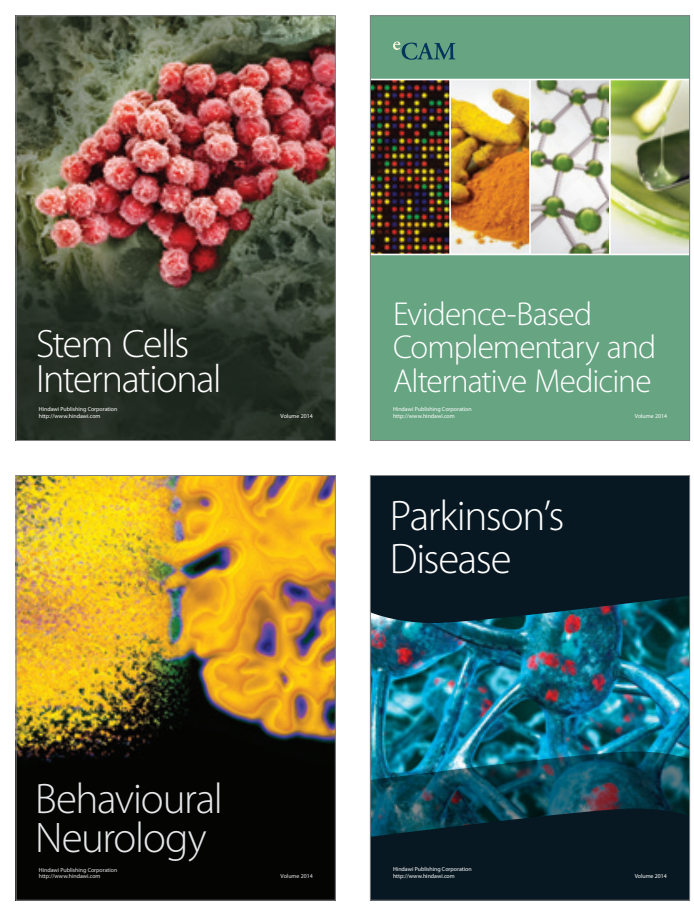

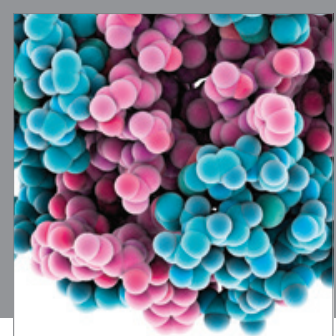

Journal of
Diabetes Research

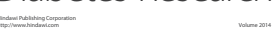

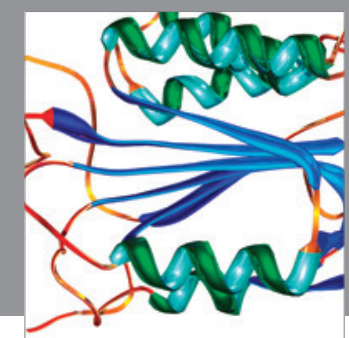

Disease Markers
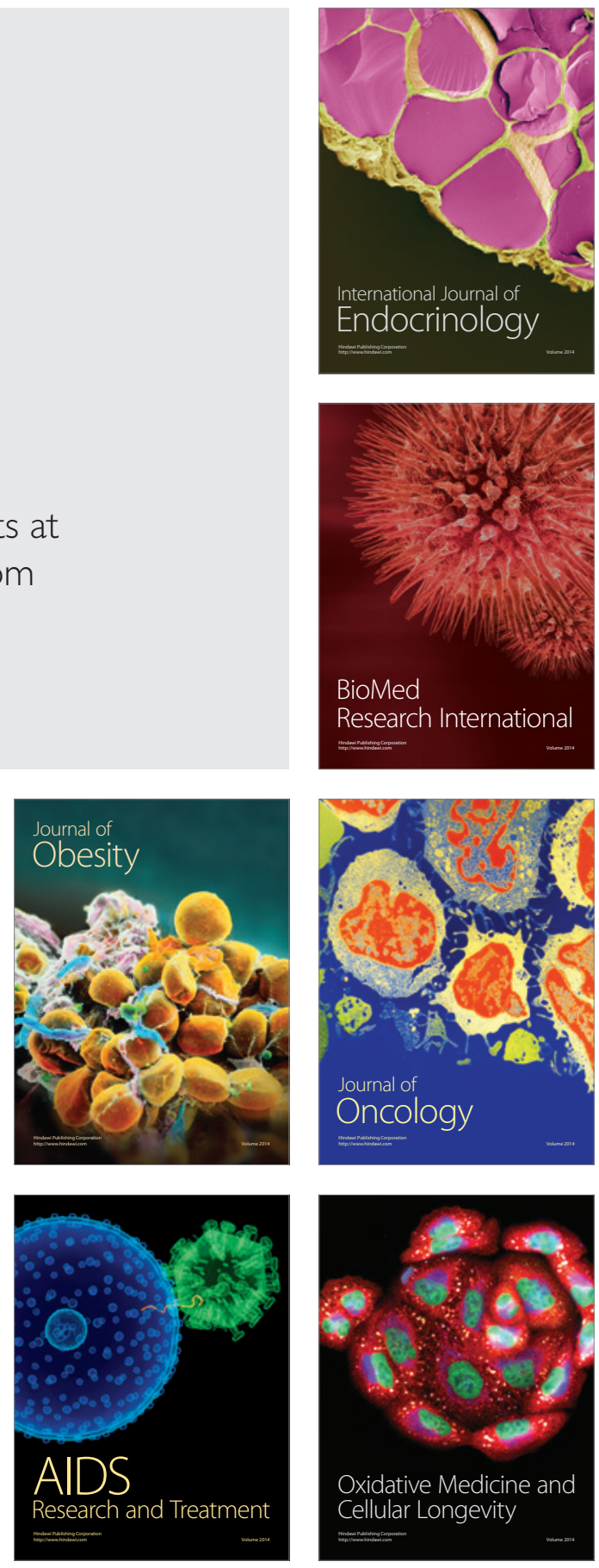